\title{
Introduction
}

\section{Cardiovascular risk: a UK priority-it's time to act II}

C ardiovascular disease is the major cause of premature death and disability in the UK, and is a major contributor to the escalating costs of health care. In most cases, atherosclerosis is the underlying pathology. Atherosclerosis develops insidiously over many years and is usually advanced by the time that patients develop symptoms; death, myocardial infarction, and stroke frequently occur suddenly, often before patients can access medical care.

Therefore the case for adopting an early preventive approach is a strong one. Fortunately, it is well established that the occurrence of cardiovascular disease relates strongly to lifestyle and modifiable physiological factors. Modification of these risk factors has been unequivocally shown to impact upon mortality and morbidity from cardiovascular disease.

However, treating individual risk factors in isolation is of little use; it is only by taking a global approach to cardiovascular risk management that we can effectively reduce premature cardiovascular death. This is reflected in both national and international guidelines, which in recent years have advocated tailoring prevention and treatment approaches to the individual patient's risk profile.

Depending upon the patient's risk profile, the management focus will be on lifestyle modification and the treatment of hypertension, lipid disorders, diabetes or other co-morbid conditions-for which we can call upon a growing range of highly effective drugs.

In recent years, UK doctors, nurses, and pharmacists have made great strides in tackling cardiovascular risk factors such as high blood pressure, high serum cholesterol, and diabetes. Unfortunately, however, changing patterns of behaviour that patients have spent many years developingunhealthy diets, smoking, sedentary lifestyles - still pose a major challenge.

Many people find it difficult to change their lifestyle when advised to do so by a health care professional. This is particularly the case for those who are socially or economically disadvantaged, for those who have little control over their working activities, and those who live in stressful family situations or lack social support. Furthermore, negative emotions such as depression or hostility may pose further barriers to the preventive efforts of health care professionals.

"Cardiovascular risk: a UK priorityit's time to act II", was the title of a one day conference held at the Royal College of Physicians on 14 November 2003. It was the second in an ongoing series of meetings that sought to promote wide ranging discussion of these and other issues relevant to the prevention and management of cardiovascular disease.

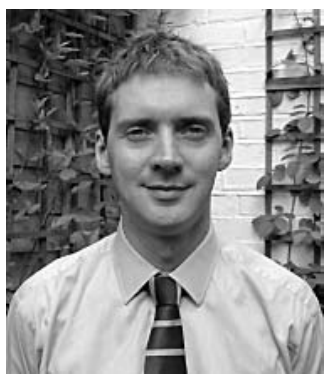

The meeting began with an overview by Professor Roger Boyle, National Heart Director, of current targets and progress towards meeting them. Subsequent speakers included practising doctors, health psychologists, nurses, and pharmacists, all with extensive experience in day-to-day patient management. They shared their experiences of running cardiovascular prevention clinics, working with patients, and of managing the cost of this work.

As health professionals we all have much to learn from each other through sharing our experiences of clinical practice. This supplement contains papers based on the presentations made at the meeting and the discussions that they prompted.

Martin R Cowie, Guest Editor Clinical Cardiology, National Heart and Lung Institute, Faculty of Medicine, Imperial College, Dovehouse Street, London SW3 6LY, UK' m.cowie@imperial.ac.uk

This supplement has been produced with the support of an unrestricted educational grant from Pfizer Ltd. The views expressed are those of the individual authors, and may or may not represent the views of Pfizer Ltd 